Ankara Üniversitesi

SBF Dergisi,

Cilt 66, No. 4, 2011, s. 125 - 152

\title{
SURVOL DE LA LITTÉRATURE SUR LES MODÈLES DE TAUX DE CHANGE D’ÉQUILIBRE: ASPECTS THÉORIQUES ET DISCUSSIONS GOMPARATIUES
}

\author{
Dr. Akm Usupheyli \\ Ankara Üniversitesi \\ Siyasal Bilgiler Fakültesi
}

\section{Résumé}

Les déviations des taux de change réel de son taux d'équilibre sont considérées comme l'une des raisons les plus importantes de pertes de compétitivité d'un pays, d'une mauvaise répartition des sources entre les secteurs, des crises de change et es déséquilibres macroéconomiques mondiaux. Par conséquent, la définition et le calcul d'un taux de change réel d'équilibre deviennent un sujet important en macroéconomie. Comme la littérature récente sur le taux de change d'équilibre montrent que la parité de pouvoir d'achat (PPA) n'est pas une méthode appropriée pour définir un taux de change d'équilibre, un certain nombre d'économistes avaient fait tourner leurs attentions vers les modèles d'équilibre du taux de change dans lequel les véritables déterminants de l'échange taux sont explicitement déterminés. Le but de ce papier est de présenter un aperçu critique de «la plus couramment appliquée» modèles théoriques et empiriques des taux de change d'équilibre. Nous, premièrement, examinons les concepts sous-jacents, les hypothèses et les bases analytiques de ces modèles. A la fin, nous allons nous concentrer sur la discussion des forces et des insuffisances de chaque modèle.

Mots clés: Taux de change d'équilibre, deviation du taux d'équilibre, FEER, BEER, NATREX

Denge döviz kuru modelleri üzerine bir literatür taramasi: teorik görünüm ve karşılaştırmalı tartışma

\section{Özet}

Reel döviz kurunun denge düzeyinden sapması, bir ülkenin rekabet gücündeki kayıpların, kaynakların sektörler arasında dengesiz dağılımının, döviz krizlerinin ve küresel makroekonomik dengesizliklerin en önemli nedenleri arasında kabul edilmektedir. Bu sebeple bir denge döviz kuru belirlemek ve hesaplamak makroekonominin önemli konularından biri haline gelmiştir. Denge döviz kuru üzerine olan en son çalışmaların satın alma gücü paritesinin denge döviz kurunu belirlemek için uygun bir yöntem olmadığını göstermesi nedeniyle, birçok iktisatçı dikkatlerini döviz kurunun reel belirleyenlerinin açıkça ortaya konduğu denge döviz kuru modellerine çevirmişlerdir. Bu makalenin amacı, en sık kullanılan teorik ve ampirik denge döviz kuru modellerine eleştirel bir bakış sunmaktır. İlk olarak bu modellerin temel kavramlarını, varsayımlarını ve analitik temellerini incelenmektedir. Makalenin sonunda ise, her modelin güçlü ve zayıf yönlerinin tartışılması üzerinde durulacaktır.

Anahtar Sözcükler: Denge döviz kuru, dengeden sapma, FEER, BEER, NATREX 


\section{Survol de la Litterature Sur les Modèles de Taux de Change D'équilibre: Aspects Theoriques et Discussions Comparatives}

\section{Introduction}

L'émergence du régime de change flottant, après l'effondrement du système de Bretton Woods, a causé des fluctuations des taux de change réels. Ces fluctuations ont eu des effets macro-économiques indésirables sur les économies. Les prix nominaux du pétrole et de la majorité des dettes internationales étant libellés en dollar, les fluctuations étaient capables de désordonner les marchés. Elles ont alors lourdement pesé sur le niveau et la structure de l'activité productive des pays. Ces problèmes ont provoqué des crises financières ayant des conséquences destructrices sur le chômage, sur l'allocation des ressources, sur le secteur réel et sur le moral des sociétés.

L'analyse traditionnelle considérait le taux de change comme le reflet de l'état de la balance courante du pays émetteur de la monnaie. Cependant, après le développement des marchés internationaux de capitaux, la libéralisation financière et la suppression des contrôles des changes au début des années 80 ont renversé les schémas d'analyse traditionnelle des marchés des changes. L'observation des taux de change effectifs à moyen et à long termes montre que les écarts se creusent entre pays, certains connaissant une tendance à l'appréciation de leur monnaie par rapport à celles de leurs partenaires commerciaux et d'autres à la dépréciation.

Sachant que le taux de change d'équilibre est supposé représenter le bon fonctionnement de l'économie, une meilleure connaissance des mécanismes déterminants le taux de change d'équilibre est devenue un indicateur essentiel pour les policy makers. En somme, l'efficacité du taux de change en tant 
qu'instrument de la politique économique dépend fortement de la connaissance de son niveau d'équilibre.

La volatilité observée des taux de change réels a conduit les économistes, notamment après les années 80 , à rechercher les déterminants d'un niveau d'équilibre pour le taux de change réel. La théorie de référence en termes de détermination du taux de change d'équilibre est la théorie de la parité des pouvoirs d'achat. La PPA, enoncée pour la première fois par Cassel (1916), il y a trois quarts de siècle, est considérée comme le «bon » prix relatif entre deux pays, autrement dit le bon niveau de change. Elle stipule que le taux de change varie de façon à compenser les différences d'inflation entre les économies concernées. Pourtant, face aux insuffisances de la théorie de la PPA, des approches alternatives ont été développées pour définir le taux de change réel d'équilibre.

Il s'agit donc de calculer le taux de change réel permettant d'obtenir une valeur cible de compte courant "structurel", une fois éliminées les influences "conjoncturelles" de l'activité domestique et étrangère sur le solde extérieur. Puisque le modèle vise à calculer le taux de change d'équilibre en s'appuyant sur les conditions économiques fondamentales qui persistent à moyen terme, il ne tient pas compte des cycles de court terme et des mouvements conjoncturels. L'une des caractéristiques principales du FEER (Fundamental Equilibrium Exchange Rate) est qu'il s'agit d'un modèle de moyen terme.

Borowski et Couharde (1999) soulignent que l'approche de Williamson est une approche en termes de statique comparative. Elle identifie à chaque période le mésalignement réel induit par les déséquilibres internes et externes en ignorant les modalités du retour du taux de change à son niveau d'équilibre.

Afin de dépasser cette faiblesse, Artis et Taylor (1993) ont proposé le concept de taux de change d'équilibre désiré DEER (Desired Equilibrium Exchange Rate). Bien que présentant beaucoup de similitudes, la différence entre le FEER et le DEER est que l'équilibre externe, dans le modèle DEER, est défini en termes de politique optimale. Autrement dit, la cible de compte courant doit être en conformité avec les politiques appliquées par les policymakers qui les considèrent optimaux. Cette terminologie n'implique pas que le DEER calculé est celui qu'on a souhaité mais c'est le taux issu de la réalisation des positions « désirées » des équilibres externe et interne.

Il existe trois facteurs influençant le FEER : Ces facteurs suggèrent que le FEER décrive une trajectoire plutôt qu'une valeur constante.

Le premier facteur est la différence de taux de croissance entre les pays. Balassa (1964) montre que la productivité dans les secteurs des biens échangeables croît beaucoup plus vite que celle dans les secteurs des biens nonéchangeables et pour cette raison, elle est plus grande dans les pays ayant un 
taux de croissance élevé. Ainsi, si nous calculons les taux d'inflation en utilisant un indice de prix couvrant les deux secteurs, les taux de l'inflation calculés qui sont égaux pourraient correspondre à une compétitivité croissante $\mathrm{du}$ secteur des biens échangeables dans les pays ayant une croissance plus grande et qui nécessite une appréciation réelle de leurs monnaies.

Le deuxième facteur est le déficit de la balance courante. Les déficits augmentent les dettes extérieures nettes. Afin de maintenir les balances du compte courant, une dépréciation réelle de monnaie est alors nécessaire pour le financement des paiements des intérêts de ces dettes en améliorant la balance commerciale.

Quant au troisième et dernier facteur, si le produit de l'élasticité revenu de la demande d'importation et du taux de croissance domestique dépasse celui entre de l'élasticité revenu de la demande d'exportation et du taux de croissance $\mathrm{du}$ tiers monde, nous observons une détérioration du compte courant (Houthaker et Magee 1969). Il est probable que cette situation provoque une dépréciation. Krugman (1989) affirme que même si ces rapports sont égaux, s'il existe une divergence importante initiale entre les niveaux d'exportation et d'importation, une dépréciation du taux de change serait alors observée. Williamson (1994 : 182) appelle ce phénomène le "gap factor".

Il faut aussi ajouter qu'il existe des facteurs exogènes (comme la chute ou la hausse des prix du pétrole) qui sont capables d'engendrer des modifications du FEER. En outre, un changement observé du FEER peut provoquer une variation sur des investissements directs étrangers via les coûts relatifs de production, dont, en particulier, les coûts relatifs de travail. Par exemple, un FEER faible augmentera l'exportation nette, mais il peut également engendrer un afflux de capitaux lié à l'augmentation des investissements directs. Si nous ne prenons en considération que le premier effet, en supposent les investissements directs exogènes, nous risquons de surestimer le FEER. Borowski et Couharde (1999) ajoutent aussi que les politiques qui affectent le niveau d'investissement peuvent modifier la croissance potentielle et donc le niveau d'activité à moyen terme.

Wren Lewis et Driver (1998) soulignent d'autre part que la politique fiscale joue un rôle important dans les calculs du FEER :

"Une diminution des dépenses publiques entraîne une diminution directe de la demande de production intérieure, tendant à causer une dépréciation du FEER. A contrario, une diminution des impôts (qui sera finalement introduite si la réduction des dépenses publiques perdure) entraînera une augmentation de la consommation, ce qui dynamisera la demande de production intérieure. Ces deux effets tireront les taux dans des directions opposées, et il est impossible de dire à priori lequel sera ea plus fort." 
Il en résulte qu'il existe une interdépendance entre les politiques budgétaires des gouvernements et le FEER.

D'après les analyses de ces facteurs et des exemples spécifiques, il est certain que le taux de change réel compatible avec l'équilibre macroéconomique peut changer au cours du temps. Il semble nécessaire de prendre en considération la trajectoire suivie du taux de change vers son équilibre et cette nécessité est soulignée par des économistes, comme Bayoumi, Clark et. al. (1994 :22), Wren Lewis (1992 : 76).

Malgré son importance, pour les policymakers, le taux de change d'équilibre fondamental n'est pas un instrument de politique économique qu'il s'agirait de manipuler pour retrouver les équilibres interne et externe. Le FEER analyse la dynamique de change de moyen terme en faisant explicitement référence aux déterminants de taux de change, et en particulier aux déséquilibres de la balance courante et aux écarts de production. Il paraît plutôt comme une variable endogène. Il revient à la politique économique (politique budgétaire et politique monétaire) de résorber l'écart à l'équilibre identifié.

Nous passons maintenant le calcul analytique du taux de change réel d'équilibre. Il s'agit de deux types de modèle. Le premier modèle considère l'équilibre interne comme exogène. Autrement dit, le taux de change réel n'a aucun impact sur la production potentielle. En revanche, dans le modèle complet, l'équilibre interne est endogénéisé en utilisant le taux de chômage d'équilibre. ${ }^{1}$

Selon le modèle partiel, formellement, le compte courant (CA) est une fonction de l'activité domestique $(\mathrm{Y})$, de l'activité étrangère $\left(\mathrm{Y}^{*}\right)$, du change réel $(\mathrm{R})$ et d'autres variables exogènes $(\mathrm{Z})$.

$$
\mathrm{CA}=f\left(\mathrm{Y}, \mathrm{Y}^{*}, \mathrm{R}, \mathrm{Z}\right)
$$

Alors, le taux de change d'équilibre $\mathrm{R}$ s'exprime par l'équation suivant ;

$$
\mathrm{C} \tilde{\mathrm{A}}=f\left(\mathrm{Y} \square, \mathrm{Y} \square^{*}, \check{\mathrm{R}}, \mathrm{Z}\right)
$$

(3)

1 Ici, nous allons détailler uniquement le modèle partiel. Pour le modèle complet, voir Usupbeyli (2010). 
où $\mathrm{C} \tilde{\mathrm{A}}, \mathrm{Y}, \square \mathrm{Y} \square^{*}, \check{\mathrm{R}}$ représentent respectivement les valeurs d'équilibre du compte courant, du PIB domestique, du PIB étranger et du taux de change réel. Ainsi, il faut trouver le taux de change réel qui permet d'obtenir une valeur du compte courant structurel en éliminant toutes les influences conjoncturelles de l'activité domestique et étrangère sur les soldes extérieurs.

La forme de la fonction $f$ est pratiquement difficile à évaluer et les variables exogènes $(Z)$ délicates à préciser. L'évaluation du change réel d'équilibre s'effectue donc en log-linéarisant le système pour déterminer l'écart à l'équilibre.

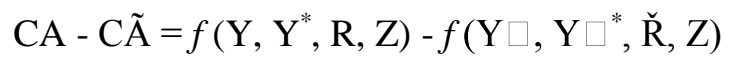

Tout d'abord, nous modélisons le commerce extérieur d'un pays donné à l'aide de deux équations de comportement en suivant Borowski, Couharde et al. (1998) :

$$
\begin{array}{ll}
\mathrm{X}=X_{o} Y_{d}^{\eta_{x}} R^{\varepsilon_{x}} & \text { exportation en volume } \\
\mathrm{M}=M_{o} Y^{\eta_{m}} R^{-\varepsilon_{m}} & \text { importation en volume } \\
\mathrm{CA}=\mathrm{PX}-\mathrm{PRM} & \text { balance courante en valeur } \\
\tau=\frac{P X}{P R M} & \text { taux de couverture }
\end{array}
$$

avec :

$Y_{d}$ : demande mondiale en volume

$\mathrm{Y}$ : demande interne en volume

$\mathrm{R}$ : taux de change réel

$P$ : prix intérieur (exogène)

En écrivant (5) et (6) en variations logarithmiques, on obtient :

$$
\begin{aligned}
& x=\frac{d X}{X}=\eta_{x} y_{d}+\varepsilon_{x} \mathrm{r} \\
& m=\frac{d M}{M}=\eta_{m} y-\varepsilon_{m} \mathrm{r}
\end{aligned}
$$




$$
\text { où } \quad y_{d=} \frac{d Y_{d}}{Y_{d}}, y=\frac{d Y}{Y} \text { et } r=\frac{d R}{R}
$$

En différenciant l'équation de la balance courante en valeur et en divisant par PRM, nous obtenons :

$$
\frac{d C A}{P R M}=\tau \frac{d X}{X}-\frac{d R}{R}-\frac{d M}{M}=\tau x-r-m
$$

Puis, en remplaçant $\mathrm{x}$ et $\mathrm{m}$ dans la relation (11) par leurs expressions respectives données par (9) et (10), nous obtenons l'expression de l'écart du taux de change à sa valeur d'équilibre (r).

$$
r=\frac{\eta_{m} y+\frac{1}{\mu} c a-\tau \eta_{x} y_{d}}{\tau \varepsilon_{x}+\varepsilon_{m}-1}
$$

\section{L’approche économétrique: le taux de change d'équilibre comportemental (BEER)}

Mac Donald (1997) et Clark et MacDonald (1999 : 291) introduisent une approche comportementaliste ; le Taux de Change d'Equilibre Comportemental (Behavioural Equilibrium Exchange Rate BEER). Le modèle BEER ne vise pas à expliquer théoriquement la détermination du taux de change réel mais à rendre compte empiriquement de son évolution en employant une technique qui étudie les mouvements du taux de change. Dufrenot, Lardic, Mathieu, Mignon et Peguin-Feissolle (2006) montrent que l'approche BEER consiste à estimer une équation réduite qui exprime le comportement du taux de change en fonction de facteurs économiques explicatifs.

Le modèle s'appuie sur les modèles alternatifs de taux de change d'équilibre pour choisir les déterminants du taux de change non seulement à moyen terme mais aussi à long terme, en évitant de fixer des contraintes a priori. Selon Dufrenot et al. (2006), le modèle BEER nous permet d'expliquer non seulement la trajectoire de long terme du taux de change, mais également les mouvements cycliques de plus court terme. 
Le BEER constitue la référence la moins normative car il repose sur l'évolution observée (et non souhaitée) du taux de change et de ses déterminants sur une longue période. La mise en place de telles relations fait donc appel à la théorie de la cointégration ainsi qu'à l'estimation des modèles à correction d'erreurs (MCE).

Clark et MacDonald (1999 : 291) proposent d'estimer tout d'abord une équation réduite, qui inclut l'ensemble des facteurs économiques fondamentaux suggérés par la théorie économique (comme le terme de l'échange, la productivité du travail, le prix du pétrole, le stock d'actifs étrangers nets, le taux de chômage, etc.) sur un panel de pays. Les auteurs testent ensuite des relations de cointégration entre le taux de change et ces variables.

$$
q_{t}=\beta_{1}^{\prime} Z_{1 t}+\beta_{2}^{\prime} Z_{2 t}+\tau^{\prime} T_{t}+\varepsilon_{t}
$$

où : $\quad \mathrm{q}_{\mathrm{t}}=\quad$ le taux de change réel courant ou observé

$\mathrm{Z}_{1}=$ un vecteur des fondamentaux économiques qui sont susceptibles d'avoir des effets persistants à long terme

$\mathrm{Z}_{2}=$ un vecteur des fondamentaux économiques qui affectent le taux de change réel à moyen terme, qui peut coïncider avec le cycle économique.

$\mathrm{T}=\quad$ un vecteur des facteurs transitoires affectant le taux de change réel à court terme.

Le taux de change réel courant est expliqué par les ensembles de variables fondamentales $Z_{1}$ et $Z_{2}$, l'ensemble de variables qui affectent le taux de change à court terme, $T$, et une erreur aléatoire $\varepsilon_{t}$. Par contre, Clark et MacDonald (1999: 292) définissent le taux de change d'équilibre comme la somme des termes relatifs aux facteurs fondamentaux de moyen et long termes.

$$
q_{t}^{\prime}=\beta_{t}^{\prime} Z_{1 t}+\beta_{2}^{\prime} Z_{2 t}
$$

Puis, ils définissent le mésalignement courant en prenant la différence entre le taux courant et le taux d'équilibre.

$$
c m_{t}=q_{t}-q_{t}^{\prime}=q_{t}-\beta_{1}^{\prime} Z_{1 t}-\beta_{2}^{\prime} Z_{2 t}=\tau^{\prime} T_{t}+\varepsilon_{t}
$$


Puisque les valeurs courantes des fondamentaux économiques ellesmêmes peuvent diverger de leur niveau souhaitable, il est également utile de définir le mésalignement total, comme la différence entre le taux réel courant et le taux d'équilibre qui dépend des fondamentaux économiques de long terme, représentés par $\bar{Z}_{1 t}$ et $\bar{Z}_{2 t}$

$$
t m_{t}=q_{t}-\beta_{1}^{\prime} \bar{Z}_{1 t}-\beta_{2}^{\prime} \bar{Z}_{2 t}
$$

Clark et MacDonald (1999: 292) décomposent ensuite le mésalignement total en deux composants, en introduisant l'équation (14) dans le membre de droite de (16)

$$
t m_{t}=\left(q_{t}-q_{t}^{\prime}\right)+\left[\beta_{1}^{\prime}\left(Z_{1 t}-\bar{Z}_{1 t}\right)+\beta_{2}^{\prime}\left(Z_{2 t}-\bar{Z}_{2 t}\right)\right]
$$

Le premier terme de l'équation (17) représente le mésalignement courant expliqué par l'équation (15). Les termes entre crochets montrent les effets de la divergence des variables économiques fondamentaux de leurs valeurs soutenables. Compte tenu de la dernière expression de $\mathrm{cm}_{t}$ dans (15), on peut aussi écrire l'équation (17) comme:

$$
t m_{t}=\tau^{\prime} T_{t}+\left[\beta_{1}^{\prime}\left(Z_{1 t}-\bar{Z}_{1 t}\right)+\beta_{2}^{\prime}\left(Z_{2 t}-\bar{Z}_{2 t}\right)\right]+\varepsilon_{t}
$$

Ainsi, Clark et MacDonald (1999 : 292) admettent que l'approche du BEER décompose le mésalignement total du taux de change en 3 éléments : les facteurs transitoires, les écarts des fondamentaux économiques à leurs valeurs d'équilibre et une perturbation aléatoire. Pour cette raison, ce modèle est plus utile pour expliquer les mouvements conjoncturels et cycliques du taux de change. Selon Driver et Westaway (2005), le BEER pourrait être défini comme le «taux de change d'équilibre courant et cyclique », puisque leur calcul est basé sur les niveaux courants et d'équilibre des facteurs fondamentaux. D'ailleurs, ils incluent également les variables cycliques qui peuvent avoir un effet persistant sur le taux de change, qui est voué à disparaître avec le temps.

Conformément à l'approche retenue par Clark et MacDonald (1999), le point de départ du modèle BEER est la condition de parité des taux d'intérêt non couverts ajustée au risque ou risk-adjusted uncovered interest rate parity (UIP) : 


$$
E_{t}\left(e_{t+1}\right)-e_{t}=\left(i_{t}-i_{t}^{*}\right)-\lambda_{t}
$$

où $E_{t}\left(e_{t+1}\right)$ est la valeur du taux de change nominal anticipée en $t$ à l'horizon $(t+1)\left(e_{t}\right.$ est le logarithme du taux de change nominal au certain), $i_{t}, i_{t}{ }^{*}$ sont les taux d'intérêts nominaux national et étranger calculés sur des titres publics d'échéance $\mathrm{n}$ et $\lambda_{\mathrm{t}}$ représente la prime de risque.

Par soustraction dans les membres de gauche et de droite du différentiel d'inflation anticipée Et $(\Delta \mathrm{pt}+1-\Delta \mathrm{p} * \mathrm{t}+1)$, nous obtenons :

$$
E_{t}\left(q_{t+1}\right)-q_{t}=r_{t}-r{ }_{t}-\lambda_{t}
$$

avec Et $\left(\mathrm{q}_{\mathrm{t}+1}\right)$ la valeur anticipée du taux de change réel en $\mathrm{t}$ pour $\mathrm{t}+1$, et $\mathrm{r}_{\mathrm{t}}=\mathrm{i}_{\mathrm{t}}-\mathrm{E}_{\mathrm{t}}\left(\Delta \mathrm{p}_{\mathrm{t}+1}\right)$ le taux d'intérêt réel national ex-ante. En réorganisant l'équation (20), nous obtenons l'expression du taux de change réel qui dépend de la valeur anticipée du taux de change réel en $t$ pour $t+1$, le différentiel de taux d'intérêt réel ex-ante et la prime de risque de change.

$$
\mathrm{q}_{\mathrm{t}}=\mathrm{E}_{\mathrm{t}}\left(\mathrm{q}_{\mathrm{t}+1}\right)-\left(\mathrm{r}_{\mathrm{t}}-\mathrm{r}_{\mathrm{t}}^{*}\right)-\lambda_{\mathrm{t}}
$$

$\mathrm{E}_{\mathrm{t}}\left(\mathrm{q}_{\mathrm{t}+1}\right)$ est supposé dépendre des fondamentaux de moyen et long termes, ainsi :

$$
\mathrm{q}_{\mathrm{t}}=\bar{q}-\left(\mathrm{r}_{\mathrm{t}}-\mathrm{r}_{\mathrm{t}}\right)-\lambda_{\mathrm{t}}
$$

où $\bar{q}$ est le vecteur des fondamentaux.

Clark et Macdonald (1999: 298) définissent la variable des fondamentaux de moyen et long termes $\left(q_{\mathrm{t}}\right)$ :

$$
\bar{q}_{\mathrm{t}}=\left(\mathrm{nfa}_{\mathrm{t}}, \text { tot }_{\mathrm{t}}, \mathrm{tnt}_{\mathrm{t}}\right)
$$

où nfa $\mathrm{a}_{\mathrm{t}}$ est $\mathrm{la}$ valeur nette des actifs extérieurs, tot $\mathrm{t}_{\mathrm{t}}$ est les termes de l'échange, tnt $_{\mathrm{t}}$ le rapport entre les prix des biens non échangeables sur les prix des biens échangeables domestiques (la variable tnt $t_{t}$ est donc une mesure de l'effet Balassa-Samuelson). Alberola, Susana, Humberto et Ubide (1999) 
proposent d'introduire les variables de coin fiscal et de rapport du déficit public au PIB domestique. MacDonald (1997) ajoute aussi le prix réel du pétrole comme une variable explicative à l'équation (23).

Meese et Rogoff (1988) invoquent que la risk premium ont une signe négative dans l'équation (21) indiquant qu'une augmentation de la prime de risk nécessite une dépréciation de taux de change réel. Clark et Macdonald (1999:298) expriment la composante de la prime de risque $\left(\lambda_{t}\right)$ comme une fonction positive du rapport entre la dette domestique et la dette étrangère du gouvernement:

$$
\lambda_{\mathrm{t}}=\mathrm{g}\left(\mathrm{gdebt} / \mathrm{gdebt}^{*}\right)
$$

Ainsi, une augmentation de la dette intérieure par rapport à la dette extérieure augmente la prime de risque domestique, ce qui nécessite une dépréciation du taux de change d'équilibre réel courant.

En combinant les équations (21)- (24), nous obtenons l'équation générale suivante :

$$
\operatorname{BEER}=\left(r_{t}-r^{*}, \text { gdebt/gdebt }^{*}, \text { nfa }_{t}, \text { tot }_{t}, \text { tnt }_{t}\right)
$$

Puisque le taux d'équilibre n'est pas une variable observable, une approche empirique commune pour estimer le modèle BEER implique deux étapes: La première étape consiste à estimer le rapport de long terme (cointégration) entre le taux de change réel et l'ensemble de fondamentaux économiques à court terme et à long terme énumérés dans l'équation

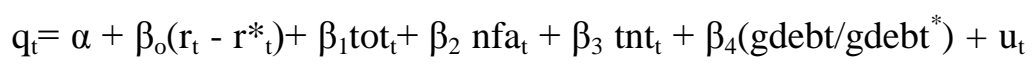

où $u_{t}$ est un processus d'erreur indépendamment et identiquement distribué (iid).

Dans une deuxième étape, on réutilise la relation de cointégration pour calculer le BEER :

$$
\mathrm{q}_{\mathrm{t}}^{\text {BEER }}=\hat{\alpha}+\hat{\beta}_{\mathrm{o}}\left(\mathrm{r}_{\mathrm{t}}-\mathrm{r}_{\mathrm{t}}{ }\right)+\hat{\beta}_{1}{ }_{1} \mathrm{tot}_{\mathrm{t}}+\hat{\beta}_{2} \mathrm{nfa}_{\mathrm{t}}+\hat{\beta}_{3} \mathrm{tnt}_{\mathrm{t}}+\hat{\beta}_{4}\left(\mathrm{gdebt} / \mathrm{gdebt}^{*}\right)
$$

Cependant, l'estimation économétrique du BEER dépend aussi des facteurs transitoires à court terme et des déviations des fondamentaux de leur 
valeur à long terme. Clark et MacDonald (2000) ont raffiné l'approche du BEER par l'introduction du concept de taux de change d'équilibre permanent (PEER). Ils utilisent la technique économétrique proposée par J. Gonzalo et Granger (1995) pour décomposer le vecteur de cointégration utilisé par le BEER en composantes permanentes et transitoires afin d'obtenir le taux de change d'équilibre permanent. Si cette décomposition est le fruit de méthodes purement statistiques, sans fondement économique explicite sur la dynamique des taux de change, comme le filtre de Beveridge et Nelson tel que pratiqué par Clark et MacDonald (2000), il s'agit de APEER (Atheoretical Permanent Equilibrium Exchange Rate). La composante permanente est considérée comme le niveau d'équilibre du taux de change soit le taux de change d'équilibre permanent. Clark et MacDonald (2000) fondent leur analyse sur le taux de change des Etats-Unis, du Canada et du Royaume-Uni. Selon leurs résultats, le PEER calcule un taux de change réel d'équilibre moins volatile que le BEER en éliminant des facteurs transitoires. Fernandez et al. (2001) calculent le taux bilatéral entre l'euro et le dollar. Égert et Halpern (2005) se concentrent sur les taux de change réel d'équilibre des pays de l'est et du centre d'Europe en utilisant le modèle PEER. Clark et MacDonald (2000) indiquent que les estimations du PEER montrent les éléments permanents du mésalignement courant. Ainsi, le modèle nous permet de séparer les variables qui déterminent la trajectoire du taux de change d'équilibre et les variables qui influencent cette trajectoire. Clark et Macdonald (2000) précisent que cette distinction est très importante pour construire les politiques économiques. Néanmoins, comme le suggèrent Driver \& Westaway (2005), cette approche demeure plus statistique qu'économiquement fondée.

Le modèle BEER a une perspective purement économétrique : il s'agit d'introduire des variables fondamentales susceptibles de comprendre des mouvements de taux de change, sans expliciter la théorie économique sousjacente. Leur interaction est analysée empiriquement en appliquant des techniques standards de cointégration. Le modèle est donc ad hoc, puisque le choix des variables $Z$ et $T$ est arbitraire.

Bouveret et Sterdyniak (2005) affirment que cette approche n'incorpore pas explicitement la dynamique du taux de change. Lorsqu'il y a un mésalignement, les fondamentaux de la relation de long terme sont censés exercer une force de rappel sur le taux de change courant pour le faire converger vers sa valeur d'équilibre. Pourtant, cette convergence provient du mécanisme statistique (le modèle à correction d'erreur) sous-jacent et non du modèle théorique. Le MCE suppose implicitement que le taux de change réel converge de façon monotone vers sa valeur de long terme. L'utilisation d'un MCE peut être justifiée par des raisons économétriques tant qu'il existe une relation de cointégration entre le taux de change réel et les fondamentaux. Or, 
selon l'analyse théorique, cette propriété n'est obtenue que dans des cas très particuliers (il faut qu'il n'y ait qu'une seule variable rigide dans l'économie, voir Féroldi et Sterdyniak, 1984). Il existe une substitution entre les concepts économiques et économétriques. Par exemple, dans l'analyse réalisée par MCE, l'équilibre de long terme, en tant que concept économique, est représenté par la relation de cointégration qui est un concept économétrique. En plus, le mésalignement du taux de change est représenté par le terme d'erreur du MCE. Dans ce contexte, soit nous ne pouvons pas obtenir une relation de cointégration avec les «vraies» fondamentaux et ne pouvons pas estimer un BEER, soit nous aurons une relation de cointégration avec d'autres fondamentaux (ou bien nous les inventerons afin d'en obtenir une) et le BEER estimé va être considéré comme le taux de change réel d'équilibre, ce qui n'est évidemment pas le cas.

En outre, Les dynamiques des taux de change dans le BEER sont en contradiction avec les dynamiques des taux de change dans les modèles économiques. Dans les modèles macro-économiques, la dynamique des taux de change dépend des anticipations et de l'ajustement lent des variables nominale et réelle comme les prix ou la position nette d'actifs étrangers. Dans ce contexte, Féroldi et Sterdyniak (1984) démontrent que s'il existe au moins deux variables prédéterminées dans l'économie, la convergence du système après un choc n'est plus monotone et, le taux de change converge vers son équilibre. Par conséquent, la dynamique des taux de change ne peut pas être réduite à un simple paramètre de correction d'erreur comme dans les modèles BEER. Ainsi, Bouveret et Sterdyniak (2005) précisent que les performances empiriques du BEER sont à juger à l'aune de l'absence d'un modèle théorique explicite, ce qui en limite la rigueur.

Le passage entre l'équation théorique et l'équation estimée suppose un grand nombre d'hypothèses. En particulier, l'équation estimée suppose implicitement un ajustement simultané du taux de change nominal, celui des prix et celui des actifs, dont les vitesses sont a priori très différentes. L'équation teste en même temps une pluralité d'hypothèses (parité non couverte des taux d'intérêt, parité des pouvoirs d'achats, relation de Fisher, etc.). Le fondement économique de la relation de long terme estimée est négligé et il s'agit d'une relation statistique.

\section{L'approche dynamique: le taux de change réel naturel (NATREX)}

Développé dans une série d'articles de Stein (1994, 1995, 2002), le taux de change réel naturel (NATREX) vise à expliquer la dynamique du taux de change réel de moyen à long terme. Il utilise le mot «naturel » pour exprimer 
le taux de change déflaté des taux d'inflation, des mouvements spéculatifs et des interventions des banques centrales. Il est un modèle d'optimisation fondé sur les comportements d'épargne et d'investissement des agents. Ce n'est pas un modèle à une équation mais plutôt un groupe de modèles chacun conçu en fonction des caractéristiques de l'économie à l'étude. Le NATREX peut être considéré comme la forme réduite de l'équation de taux de change d'un modèle macroéconométrique.

Le modèle NATREX reprend la même idée du FEER, selon laquelle le taux de change permet au compte courant d'atteindre une valeur "normale", l'activité étant à son niveau de plein emploi, mais évite de se prononcer sur le caractère "normal" du compte courant. Par ailleurs, le NATREX suppose que les agents sont incapables d'anticiper les variations du change. Le NATREX suppose que les agents forment des anticipations naïves. Cette hypothèse est certes restrictive, car la littérature montre sur données d'enquêtes que les agents forment leurs anticipations en utilisant des processus mixtes extrapolatifs, régressifs et adaptifs. (Prat et Uctum (2007))

Allen (1995:9) distingue dans le modèle NATREX trois horizons de taux de change : le court terme, le moyen terme sur lequel l'intensité capitalistique et la dette sont données, et le long terme, sur lequel elles varient. À court terme le taux de change réel dépend des fondamentaux $(Z)$, du stock d'actifs nets $(a)$ et de facteurs cycliques et spéculatifs $(c)$. Soit:

$$
q_{t}^{c}=q_{t}^{c}(\mathrm{z}, \mathrm{a}, \mathrm{c})
$$

Le NATREX de moyen terme ne dépend que des facteurs fondamentaux et du stock d'actifs nets:

$$
q_{t}^{m}=q_{t}^{m}(\mathrm{z}, \mathrm{a})
$$

Dans l'état stationnaire, le NATREX de long terme ne dépend que des fondamentaux :

$$
q_{t}^{l}=q_{t}^{l}(\mathrm{z})
$$

Selon Faruqee (1994), le NATREX porte un caractère intercyclique qui assure l'équilibre de la balance des paiements en excluant les facteurs cycliques, les flux de capitaux spéculatifs et les variations des réserves internationales. En d'autres termes, le NATREX est le taux de change réel 
d'équilibre lorsque tous les facteurs conjoncturels sont exclus et que le PNB est à sa capacité maximale. Une perturbation sur les fondamentaux du système économique pousse le taux de change d'équilibre sur une nouvelle trajectoire de moyen-à-long terme. Comme les facteurs cycliques, transitoires et spéculatifs sont considérés comme «bruit », ayant la moyenne zéro à long terme, le taux de change réel converge à la trajectoire d'équilibre. La théorie de la PPA s'avère être seulement un cas particulier de l'approche de NATREX : Selon Stein (1995 : 43-44) "le problème n'est pas de savoir si le taux de change réel est stationnaire sur une période arbitraire, mais s'il reflète les fondamentaux économiques."

Le modèle NATREX suppose la neutralité de la monnaie, en se référant l'égalité entre l'excès d'épargne sur l'investissement et le compte courant d'une part et l'hypothèse que la politique monétaire maintient l'inflation à un niveau compatible avec l'équilibre interne d'autre part (au pire à long terme). Par conséquent, le modèle se concentre sur le marché réel et le marché monétaire n'a pas besoin d'être modélisé.

Puisqu'il définit un concept d'équilibre, le NATREX devrait garantir l'équilibre interne et externe à long terme. L'équilibre interne est réalisé quand le PNB est à son niveau potentiel. L'équilibre externe est réalisé quand les comptes de la balance des paiements sont en équilibre.

Tout d'abord, le taux de change réel s'ajuste de façon à égaliser l'écart épargne-investissement $(\mathrm{S}-\mathrm{I})$ ex-ante au solde des paiements courants (CA) :

$$
\mathrm{S}-\mathrm{I}=\mathrm{CA}
$$

Ce qui implique :

$\mathrm{I}-\mathrm{S}=\mathrm{LTK}$

$\mathrm{LTK}+\mathrm{CA}=0$

LTK représentant le solde des mouvements de capitaux à long terme, qui compensent donc le déficit des comptes courants à l'équilibre.

Les mouvements des capitaux spéculatifs et les changements dans les réserves officielles sont négligés. Le modèle se concentre sur les investissements de portefeuille à long terme et les investissements directs étrangers. Ces flux capitaux nets, qui sont non spéculatifs, sont déterminés par la différence entre l'épargne et investissement national. Ainsi, le compte de capital est déterminé par (S-I) qui dépend de l'intensité capitalistique $\mathrm{k}_{\mathrm{t}}$, de la dette $F_{t}$ et des fondamentaux exogènes $Z_{t}$, que sont les préférences pour le 
présent des agents, ainsi que les termes de l'échange qui est un déterminant important du taux $q$ de Tobin et le taux de change réel.

Par conséquent, si l'on suit le modèle de NATREX, l'apparition d'un besoin de financement interne va entraîner un ajustement du taux de change réel $(\mathrm{R})$, ce qui va pousser le solde des comptes courants $(\mathrm{CA})$ à s'égaliser à l'écart (S-I) produite par une variation des fondamentaux.

$$
\mathrm{Z} \rightarrow(\mathrm{I}-\mathrm{S})(\mathrm{k}, \mathrm{F} ; \mathrm{Z}) \rightarrow \mathrm{LTK} \rightarrow \mathrm{CA}(\mathrm{R}, \mathrm{k}, \mathrm{F} ; \mathrm{Z})
$$

Ainsi l'écart (I-S) peut être financé par un excédent des mouvements de capitaux à long terme. Selon ce mécanisme, on peut clairement constater que le modèle NATREX est un modèle "self - equilibrating " d'où le qualificatif « naturel » se trouvant dans le nom du modèle :

Nous pouvons maintenant définir le solde des comptes courants :

$$
\mathrm{CA}=\mathrm{BC}-\mathrm{r}^{*} \mathrm{~F}
$$

où $\mathrm{BC}$ est le solde de la balance des biens et services, $\mathrm{r}^{*}$ est le taux d'intérêt étranger de long terme et $\mathrm{F}$ est la dette externe. Le second terme représente donc les paiements d'intérêts de la dette externe.

$\mathrm{BC}$ est l'exportation moins l'importation. Elle est une fonction négative des importations, fonction de la richesse des agents, définie comme le capital diminué de la dette externe. Elle est une fonction positive des exportations, fonction du $\mathrm{R}$ et de la demande mondiale, exogène. Alors, la balance courante dépend du $\mathrm{R}$, des fondamentaux étrangers (qui déterminent l'exportation), de la richesse domestique et les fondamentaux domestiques (qui déterminent l'importation).

La condition pour que la balance des capitaux de court terme soit équilibrée est, en émettant l'hypothèse d'égalité du risque entre les pays, que le taux d'intérêt réel domestique converge vers le taux d'intérêt réel étranger :

$$
r=r^{*}
$$

avec $r$ le taux d'intérêt réel domestique et $r^{*}$ le taux d'intérêt réel étranger. Selon cette équation, il n'existe pas une prime de risque.

Enfin, nous nous situons à l'équilibre en production de plein emploi (y): 


$$
\mathrm{Y}=\mathrm{y}(\mathrm{k}, \mathrm{Z})
$$

Le taux de variation de l'intensité capitalistique est:

$$
\mathrm{dk} / \mathrm{dt}=\mathrm{J}(\mathrm{k}, \mathrm{r}, \mathrm{Z})
$$

Le taux d'investissement est donc corrélé positivement aux fondamentaux réels (niveau et diffusion du progrès technique, prix exogène des consommations intermédiaires importées), et négativement à l'intensité capitalistique $k$ (il s'agit d'un effet de contrôle visant à assurer la stabilité de la fonction d'investissement : plus l'intensité capitalistique est forte, plus le taux d'investissement sera faible).

Nous exprimons maintenant les flux entrants de capitaux et le taux de variation de l'intensité de la dette.

Tout d'abord, nous avons:

$$
\mathrm{dF}(\mathrm{t}) / \mathrm{dt}=-\mathrm{CA}=\mathrm{LTK}=\mathrm{I}-\mathrm{S}
$$

Nous pouvons maintenant introduire la fonction d'épargne, qui fournit une contrainte budgétaire intertemporelle pour que $\mathrm{F}(\mathrm{t})$ se stabilise à une valeur $\mathrm{F}^{*}(\mathrm{t})$. Quand la dette est stabilisée, c'est à dire que nous avons $\mathrm{F}(\mathrm{t})=$ $\mathrm{F}^{*}(\mathrm{t}), \mathrm{CA}=0$ et nous pouvons alors écrire :

$$
\mathrm{BC}=\mathrm{r}^{*} \mathrm{~F}
$$

Le service de la dette, issu de la dette passée, doit alors être compensé par un excédent commercial, pour que $\mathrm{CA}=0$.

Lors de l'ajustement, quand I > S, F (t) augmente. Si nous voulons assurer une stabilisation de $\mathrm{F}(\mathrm{t})$ à un horizon fini, afin d'éviter une explosion de la dette, qui rendrait le modèle instable, il faut introduire une corrélation positive entre dette et épargne : une hausse de $\mathrm{F}(\mathrm{t})$ entraîne une hausse de l'épargne. Cela s'explique par le fait que la montée de l'endettement incite le gouvernement à réduire le déficit et, à cette fin, à baisser des dépenses et dégager ainsi de l'épargne publique, ou bien par le fait que la détérioration de la situation économique mine la confiance des investisseurs et des ménages, ce qui entraîne une baisse de la consommation et de l'investissement. Ces deux 
processus vont alors induire une hausse du taux d'épargne, privée et publique. La fonction d'épargne est alors :

$$
\mathrm{S}=\mathrm{S}(\mathrm{k}, \mathrm{F}, \mathrm{Z})
$$

avec dS / dF > 0.

Il vient alors :

$$
\mathrm{I}-\mathrm{S}=\mathrm{I}(\mathrm{k}, \mathrm{Z})-\mathrm{S}(\mathrm{k}, \mathrm{F}, \mathrm{Z})=\mathrm{L}(\mathrm{k}, \mathrm{F}, \mathrm{Z})
$$

avec $\mathrm{dL} / \mathrm{dF}<0$ et $\mathrm{dL} / \mathrm{dk}<0$.

Enfin, 1'hypothèse centrale du modèle, le taux de change réel d'équilibre (TCRE) s'ajuste pour que CA s'équilibre à I - S (39) avec CA :

$$
\mathrm{CA}=-\mathrm{dF} / \mathrm{dt}=\mathrm{BC}(\mathrm{k}-\mathrm{F}, \mathrm{R}, \mathrm{Z})=\mathrm{CA}(\mathrm{R}, \mathrm{k}, \mathrm{F}, \mathrm{Z})
$$

avec $\mathrm{dCA} / \mathrm{dR}<0$, R représentant le TCRE (une hausse de R signifie, dans le cadre de ce modèle, une appréciation).

L'approche du NATREX est dynamique et repose explicitement sur les déterminants de long terme du taux de change réel d'équilibre. Contrairement au BEER, le NATREX incorpore des effets de stock à travers la dynamique de la position extérieure nette et du stock de capital. En plus, le modèle de Stein, en introduisant une fonction d'épargne agrégée corrélée positivement à la dette étrangère, permettra d'expliquer les évolutions du solde de la balance des paiements courants et de la balance des mouvements de capitaux au long terme. Ainsi, il permet de calculer une trajectoire d'équilibre du moyen terme au long terme. Néanmoins le NATREX souffre, comme le FEER, d'hypothèses restrictives sur l'équilibre interne : le marché du travail est supposé être à l'équilibre ; la dynamique d'ajustement des prix et des salaires est passée sous silence.

\section{Discussion comparative des modèles}

Bien que toutes ces approches présentent des différences significatives dans les méthodes d'évaluation du taux de change réel d'équilibre, leur 
comparaison demeure une tâche difficile. Ces méthodes recèlent encore trop d'insuffisances théoriques pour conduire à des estimations précises du taux de change réel d'équilibre et les estimations sont sans doute trop lourdes à mener dans le cadre d'une politique de benchmarking. Dans cette section, nous allons comparer les modèles en discutant leurs avantages et inconvénients aux niveaux théorique et empirique.

\subsection{Comparaison théorique}

Sur le plan théorique, l'une des critiques fréquentes adressées au FEER affirme que cette méthode ne constitue pas un véritable équilibre puisque l'équilibre de stock-flux n'est pas réalisé. Par conséquent des effets d'hystérésis apparaissent sur le FEER puisque le compte courant, à moyen terme, est affecté par des chocs provisoires (Driver et Westaway, 2005). En outre, l'analyse théorique des déterminants des taux de change réels -de même que celle de leurs mésalignements- dans le FEER s'effectue largement hors modèle, lorsque l'on étudie le solde des flux de capitaux « sous-jacents » et le niveau de production «potentiel». De plus, les effets de stocks -notamment celui de la position extérieure nette- ne sont pas pris en compte, ou le sont peu, si bien que l'analyse reste confinée au moyen terme.

MacDonald (2000) met en avant aussi la limitation analytique du modèle FEER en raison des fluctuations possibles de la valeur nette des actifs extérieurs. Supposons que, dans la période initiale, le taux de change courant soit au niveau du FEER et les équilibres internes et externes soient assurés. A la suite d'un choc exogène, par exemple une hausse des prix du pétrole, le taux de change courant se déprécie dans la période suivante, améliorant l'équilibre courant ainsi que la position de la valeur nette des actifs extérieurs. Cette dernière, alternativement, implique que dans les périodes futures le taux de change réel, conforme à l'accumulation de capital à moyen terme, ne sera plus le FEER; en particulier, le FEER doit s'apprécier pour compenser les effets de l'accumulation nette. Cet effet d'hystérésis est la conséquence inévitable d'accepter le taux de change réel comme un concept de moyen terme.

Cependant, le NATREX, contrairement au FEER, considère non seulement le moyen terme, mais analyse également le long terme, car le cadre théorique dynamique du NATREX est un cadre stock-flux. Le NATREX s'efforce d'intégrer les « effets de stocks » (dynamique de la position extérieure nette, voire du stock de capital), dont l'impact sur la dynamique de long terme des taux de change réel d'équilibre est fondamental. Alors le passage du moyen terme à l'équilibre de long terme pourrait être démontré explicitement. Ainsi, contrairement au FEER, le NATREX n'est pas récursif, car le compte de capital a un effet sur le taux de change d'équilibre de moyen et long termes. 
En comparaison avec le FEER, le NATREX analyse aussi davantage la relation entre l'évolution du taux de change réel et les comportements microéconomiques des agents. A mesure que l'épargne et l'investissement, qui sont les variables fondamentales, changent, le NATREX flotte et converge vers un taux de long terme statique. Ainsi, contrairement aux autres modèles de taux de change réel d'équilibre, le NATREX devient un taux de change d'équilibre mobile, autrement dit, le taux de change réel d'équilibre se détermine dans un cadre dynamique. Dans cette théorie, les flux de capitaux vont directement dépendre des comportements d'épargne et de consommation des différents agents économiques. Ainsi selon les préférences des différents agents, les flux des capitaux entrants et sortants vont être modifiés. De plus, dans le modèle NATREX, le niveau d'investissement dépend positivement du progrès technique. Le NATREX prend donc en compte les niveaux d'investissements et d'épargne qui entraînent un solde des flux de capitaux moyens. En conséquence, le taux de change réel est déterminé par les niveaux d'épargne, de consommation et par les progrès techniques. Le taux de change d'équilibre évolue donc dans le temps jusqu'à ce que la position extérieure nette se stabilise. Supposons un cas où il s'agit d'une hausse du niveau de consommation. Celle-ci entraîne logiquement une baisse de l'épargne des différents agents économiques qui fait monter le taux d'intérêt réel du pays. Théoriquement cette augmentation du taux d'intérêt devrait attirer les capitaux et donc faire monter le taux de change réel. Ainsi la hausse du niveau de la consommation entraîne une hausse des entrées de capitaux, une appréciation du taux de change réel et donc une dégradation du solde courant. Le modèle NATREX n'arrête pas son analyse à ce point là comme le FEER, et il continue à analyser le passage du moyen terme au long terme. Ainsi ce nouveau taux de change d'équilibre continue à se modifier: en effet, cette situation entraîne une hausse de la dette du pays du fait des taux d'intérêts plus élevés, la consommation doit donc baisser pour réduire cette dette. Cela entraîne à long terme une baisse du taux de change réel. Par conséquent, le NATREX prolonge les modèles similaires tels que la PPA et le FEER en concentrant ses analyses sur les périodes où les fondamentaux ne sont pas stationnaires et conduisent par conséquent la trajectoire du taux de change de moyen terme à la position statique de long terme.

Quant à l'approche du BEER, elle prend en considération les facteurs à court terme, contrairement au modèle FEER, qui peuvent contribuer fortement aux mouvements du taux de change réel d'équilibre du moyen au long terme. Il analyse le mésalignement total de taux de change, à un point quelconque dans le temps, en le décomposant en trois parties : des facteurs transitoires, des perturbations aléatoires et un point où les principes fondamentaux économiques divergent de leurs valeurs soutenables. Ainsi, l'estimation du BEER fournit 
aussi des informations au sujet du mésalignement courant. Ce dernier signifie un mésalignement provenant des effets transitoires et aléatoires, c'est-à-dire des facteurs non traités en tant que déterminants «fondamentaux » du taux de change (MacDonald, 2000). En particulier, l'adoption de la parité de taux d'intérêt permet à l'approche du BEER de révéler les sources des changements du compte capital qui peuvent alors également influencer le compte courant et le "comportement" du taux de change. Le BEER permet aussi de calculer un taux de change « fondamental» d'équilibre et alternativement le mésalignement total (comme dans la notion du FEER). En revanche, considérant que l'approche du FEER révèle seulement le comportement du taux de change conduit par des changements des positions des équilibres externes et internes, le BEER, plus général, peut être employé pour expliquer le mouvement cyclique du taux de change réel.

Outre son caractère statique, le FEER est critiqué aussi pour être un modèle normatif. Dans la théorie du modèle FEER, le taux de change d'équilibre représente le taux qui permet simultanément l'équilibre interne, identifié comme le niveau de la production potentiel compatible avec le plein emploi de la capacité productive et la stabilité des prix, et l'équilibre externe réalisé quand le compte courant est soutenable à long terme, autrement dit la balance courante "d'équilibre » est en égalité avec le flux de capital d'équilibre. Ainsi le modèle FEER impose des contraintes qui attribuent un caractère normatif au modèle. Tout d'abord, l'équilibre interne est supposé toujours réalisé, puisque chacun des pays utilise sa politique de change pour atteindre son objectif de balance commerciale. Autrement dit, nous avons le plein emploi de manière permanente, alors que ce n'est pas toujours le cas. En outre, la méthode du FEER nécessite la coordination des pays pour un solde courant soutenable. Par contre, il n'est pas garanti que chaque pays veuille que son taux de change soit en permanence au niveau d'équilibre. Un pays peut souhaiter surévaluer son taux de change pour réduire son inflation, ou bien le sous-évaluer pour relancer sa croissance. Surtout pour les économies émergentes la notion de plein emploi n'a pas de sens bien que ceux-ci disposent d'un stock important de travailleurs potentiels. Quant à l'équilibre externe, le FEER suppose aussi que le compte courant à l'équilibre est égal au flux net du capital privé et officiel déterminé par l'écart épargneinvestissement. Ceci implique que le taux de change d'équilibre vérifie cette égalité à moyen terme d'où la notion de balance courante d'équilibre puisque nous pouvons avoir une balance courante déficitaire mais soutenable à moyen terme. Alors, le FEER recherche une mesure du taux de change réel qui guide les politiques vers un équilibre du compte courant avec des mouvements de capitaux souhaitables. 
Outre l'aspect normatif de la mesure du compte capital soutenable, MacDonald (2000) admet que les estimations des élasticités commerciales imposent "une couche supplémentaire" au caractère normatif du modèle FEER. Siregar and Rajan (2006) supposent que selon la forme la plus simple de compte courant $(\mathrm{CA}=\mathrm{ntb}+\mathrm{nfa}$ ntb où nfa (net foreign assets) est la balance de commerce nette et ntb (net trade balance) est la valeur nette des actifs extérieurs)) où le composant ( $n f a$ ) est nul ou faible relativement à (ntb), l'élasticité du taux de change réel par rapport aux importations doit donc être proche de l'élasticité du taux de change réel par rapport aux exportations, sous l'hypothèse de "l'équilibre externe". Autrement dit, l'élasticité commerciale estimée du FEER est effectivement proche de zéro. Une dépendance importante de FFER à l'élasticité commerciale peut produire une évaluation imprécise (inaccurate) de la trajectoire du FEER. Une dépréciation du taux de change réel domestique mènerait non seulement à une amélioration de (ntb) mais aussi à une augmentation de $(n f a)$. Si le FEER mesure seulement les changements dans (ntb) et suppose que l'impact sur nfar (returns on net foreign assets) est déterminé de façon exogène, alors le taux de l'appréciation du taux de change nécessaire risque d'être surestimé. En outre, Barisone, Driver et Wren-Lewis (2006) décrivent les paiements d'intérêt des actifs nets extérieurs comme «le talon d'Achille» du modèle FEER. Par conséquent, le mésalignement estimée par le FEER est susceptible de ne pas être fiable.

Le modèle NATREX se distingue de l'approche FEER par son aspect résolument positif, tourné vers l'empirisme. Plutôt que de déterminer ce que devrait être le "bon " taux de change, il se propose d'expliquer les mouvements de moyen et de long terme effectivement observés.

Dans ce sens, le NATREX est le taux impliqué par les fondamentaux réels et par les politiques économiques existantes. Il ne s'intéresse pas à l'optimalité au niveau social de ces politiques et n'a pas l'ambition d'être le taux de change réel optimal. Le NATREX nous donne le taux d'équilibre imposé par les fondamentaux économiques. Par exemple, le NATREX prévoit les effets d'un choc exogène dans l'économie publique ou privée sur le taux de change réel d'équilibre sans considérer si c'est une perturbation désirée ou pas. Les modèles de NATREX ne portent aucun jugement par rapport à la conformité des fondamentaux à la richesse sociale. À cet égard, le NATREX diffère du FEER. Alors que le FEER représente le taux de change permettant au compte courant d'atteindre une valeur "normale" imposée de façon arbitraire en supposant que les productions domestiques et étrangères potentielles sont atteintes, les théoriciens du NATREX ne se prononcent pas sur le caractère «normal » du compte courant, mais se fondent sur des facteurs structurels comme déterminants du taux de change réel d'équilibre. Tandis que le FEER dépend des notions abstraites et subjectives comme "l'équilibre externe 
soutenable" et "l'équilibre interne", le NATREX est calculé en utilisant les niveaux courants des fondamentaux économiques. Cet élément normatif est l'une de différences principales entre la définition du NATREX et du FEER. Selon Allen (1995), bien que les politiques optimales prescrites ne font pas partie de la définition du NATREX, de telles politiques pourraient être introduites dans un modèle de NATREX pour déterminer le taux de change d'équilibre désiré.

Le BEER explique, lui aussi, le taux de change en se fondant sur différents facteurs économiques (valeurs des exportations, importations et niveau des taux d'intérêts), tout comme le FEER. Mais le BEER fait de plus une différence entre secteurs exposés et arbitrés, ce qui a pour avantage de prendre en compte des effets Balassa-Samuelson. Une autre différence principale entre le FEER et le BEER est que le BEER est estimé en utilisant des valeurs courantes des déterminants fondamentaux du taux de change réel. Alors, la théorie du BEER a pour avantage de se fonder sur l'évolution observée (et non souhaitée ou estimée) du taux de change, contrairement aux modèles du FEER qui exigent des variables non-observables générant ainsi un caractère normatif.

De surcroît, Clark et Macdonald (1999 : 317) supposent que pour le BEER, il n'est pas nécessaire d'attribuer une valeur «soutenable» pour l'équilibre externe pour deux raisons. Premièrement, le modèle repose sur la parité des taux d'intérêt non couverts et donc il n'existe aucune contrainte sur le financement d'un déséquilibre externe. Deuxièmement, le modèle contient des mécanismes d'ajustement qui permettent au taux de change réel de s'ajuster par rapport aux changements des actifs extérieurs nets et de la dette de gouvernement, de sorte que l'équilibre externe est atteint, au moins à long terme. En plus, le modèle BEER peut s'adapter aux besoins ou priorités économiques des économistes.

\subsection{Comparaison empirique}

Malgré leurs fondements théoriques assez similaires, ces approches sont considérablement différentes dans leur méthode d'estimation. L'approche du FEER est fondée sur un modèle structurel qui décrit principalement les relations de commerce extérieur. En plus, il relie explicitement les mouvements des taux de change aux déséquilibres internes et externes. Il existe essentiellement deux approches pour estimer le FEER. La première consiste à prendre un modèle macroéconométrique estimé, d'imposer les équilibres externe et interne, et de le résoudre pour trouver le taux de change réel qui correspond au FEER. En outre, le modèle macroéconométrique multinational permet d'estimer des taux de change d'équilibre des principaux partenaires 
commerciaux. Dans les modèles macro multinationaux appliqués pour estimer le FEER, où les taux de change réel d'équilibre sont déduits pour l'ensemble de pays inclus dans le modèle, on suppose que le taux de change réel du pays d'origine affecte les variables économiques des autres économies. Cependant, Macdonald (2000) affirme que les développements dans de petites économies sont susceptibles de n'avoir aucun impact sur le monde extérieur. C'est pourquoi lorsqu'on analyse le taux de change d'équilibre d'un petit pays, les modèles structurels du seul pays décrivant seulement le commerce extérieur de l'économie d'origine sont bien plus fiables.

La deuxième approche utilisée pour estimer le FEER est la plus populaire et consiste à se concentrer sur une équation de compte courant telle que $(\mathrm{CA}=\mathrm{ntb}+\mathrm{nfa})$ et de l'égaliser à un compte de capital soutenable.

En outre, le FEER s'applique dans un cadre dit de "statique comparative" c'est-à-dire qu'il consiste à examiner la distance du taux de change réel par rapport à sa valeur d'équilibre à un instant donné. La mise en œuvre de cette statique comparative nécessite la connaissance des blocs macroéconomiques du commerce extérieur, plus précisément des élasticités de commerce en volume, dont les séries sont rarement disponibles sur de longues périodes pour les pays émergents.

Bien que les applications des modèles FEER se fondent sur un modèle structurel, elles sont très coûteuses à mettre en œuvre dans la mesure où elles nécessitent soit la construction d'un modèle économétrique décrivant les échanges mondiaux (Wren-Lewis et Driver, 1998), soit l'utilisation du bloc des échanges extérieurs d'un modèle multinational (Borowski et Couharde, 1999).

Bien qu'ils présentent plusieurs limites spécifiques et que l'interprétation de leurs résultats soit moins intuitive que celle des FEERs, le NATREX et le BEER présentent l'avantage essentiel d'être fondés sur une estimation par les techniques modernes de l'économétrie des séries temporelles. Ce faisant, elles permettent de calculer une trajectoire du taux de change réel d'équilibre de long terme, voire une autre de moyen terme, à partir desquelles les mésalignements peuvent être directement quantifiés. Le NATREX a recours tout comme le BEER à une estimation du taux de change d'équilibre par une équation réduite, au moyen de relations de long terme existant entre le taux de change réel et ses fondamentaux (dans le cadre du NATREX (préférence pour le présent et progrès technique)). Les principes fondamentaux et les spécifications de l'équation réduite des modèles NATREX et BEER sont fondés sur un modèle structurel qui se concentre sur la soutenabilité de l'équilibre externe ou sur un équilibre de portefeuille.

Au sein des ces approches dynamiques, le NATREX paraît plus solide que le BEER sur le plan théorique. En effet, dans le NATREX, les 
fondamentaux théoriques du taux de change y sont clairement identifiés. L'implémentation empirique du modèle de NATREX se fonde habituellement sur l'estimation d'une relation de cointégration entre le taux de change et ces principes fondamentaux.

Cependant, le BEER se fonde sur la notion statistique de l'équilibre. L'idée générale est d'évaluer le comportement du taux de change d'équilibre par une équation réduite, ceci au moyen de relations de long terme existant entre le taux de change réel et diverses variables macroéconomiques. Celles-ci sont choisies parmi les variables que la théorie économique prescrit comme variables influençant les équilibres internes et externes et susceptibles d'avoir un rôle à jouer au court et moyen terme. Alors, dans le BEER, les fondamentaux ne sont pas identifiés à partir d'un modèle théorique consacré. L'approche du BEER modélise directement le taux de change réel d'équilibre pour des biens commerciaux en fonction notamment de la position externe nette. Dans le modèle BEER, l'endogénéite de certains régresseurs peut conduire à des biais d'endogénéite. De plus, le modèle BEER ne montre pas la nature du processus de convergence du taux de court terme/courant au taux d'équilibre (MacDonald, 2000)

Le caractère ad-hoc du choix des variables exogènes se voit également au travers des évaluations empiriques: puisque beaucoup de différentes variables peuvent être potentiellement introduites dans la relation de cointégration de long terme dans les estimations du BEER, les résultats souffrent aussi d'un manque de généralité.

Une autre limite tient à ce que le modèle BEER ne justifie pas l'origine ni la forme de la force de rappel vers la relation de long terme. Dans ce type de modèle, la force de rappel est en fait imposée par le modèle économétrique utilisé, et non par un modèle macroéconomique sous-jacent. De plus, le modèle BEER suppose que le taux de change courant converge de façon monotone vers le taux de change de long terme. Or s'il existe plus d'une source de rigidités dans l'économie (rigidité des prix et ajustement lent de la position extérieure nette par exemple), la convergence n'est plus forcément monotone et le taux de change courant peut fluctuer autour de sa valeur de long terme, ou encore converger de façon asymétrique nécessitant la mise en œuvre de modèles nonlinéaires.

\section{Conclusion}

Même si ces approches sont différentes l'une de l'autre, nous avons vu qu'il était difficile de déterminer si l'une d'elles est meilleure ou non que l'autre. L'approche du FEER présente l'avantage de reposer sur des fondements macroéconomiques solides mais elle a un caractère absolument normatif. De 
plus, elle est difficile à mettre en ouvre empiriquement. Quant à l'approche du BEER, elle ne dispose pas de fondement théorique solide. De plus, elle laisse même la détermination des variables expliquant le taux de change réel au modèle économétrique. Face à ces insuffisances théoriques du BEER et aux nombreuses difficultés empiriques soulevées par l'application du FEER, nous nous sommes tournés vers l'approche de NATREX qui présentent plusieurs avantages sur le plan à la fois théorique et à la fois empirique et qui reste toujours le modèle le plus approprié pour l'estimation de taux de change réel d'équilibre.

\section{Bibliographie}

Alberola, E., Susana, C., Humberto, L. et Ubide, A. (1999), "Global Equilibrium Exchange Rates: Euro, Dollar, 'Ins', 'Outs', and Other Major Currencies in a Panel Cointegration Framework," IMF Working Paper 99/175.

Allen R. P. (1995), "The Economic and Policy Implications of the NATREX Approach"; Stein J. et Allen P. R. (eds.), Fundamental Determinants of Exchange Rates (Oxford, Oxford University Press).

Artis, M. J. et Taylor, M. P. (1993), "DEER Hunting: Misalignment, Debt Accumulation and Desired Equilibrium Exchange Rates" IMF Working Paper : 1-36.

Balassa, B. (1964), "The Purchasing Power Parity Doctrine: A Reappraisal" Journal of Political Economy, 72 (6) : 584-596.

Barisone, G., Driver, R. L. et Wren-Lewis, S. (2006), "Are our FEERs Justified?," Journal of International Money and Finance, 25 (5) : 741-759,

Bayoumi T., Clark, P., Symansky. S. et Taylor, M. (1994), "The Robustness of Equilibrium Exchange Rate Calculations to Alternative Assumptions and Methodologies". Williamson J. (eds.), Estimating Equilibrium Exchange Rates. (Washington, Institute for International Economics.).

Borowski, D. et Couharde, C. (1999), "Quelle Parite d'Equilibre pour l'Euro?" Economie Internationale, 21-44.

Bouveret, A. et Sterdyniak H. (2005), "Les Modèles de Taux de Change d'Equilibre de Long Terme, Dynamique et Hystérèse." Revue de l'OFCE, 93: 245-286.

Cassel, G. (1916), “The Present Situation of the Foreign Exchange”. Economic Journal 26: 62-75.

Clark, P. B., Bartolini, L., Bayoumi, T. et Symansky, S., (1994), "Exchange Rates and Economic Fundamentals: a Framework for Analysis," IMF Occasional Paper, 115. 
Clark, P.B. et R. MacDonald, (1999), "Exchange Rates and Economic Fundamentals: A Methodological Comparison of BEERs and FEERs" MacDonald R. and Stein J (eds.) Equilibrium Exchange Rates, (Amsterdam, Kluwer).

Clark, P.B. et MacDonald, R. (2000), "Filtering the BEER: A Permanent and Transitory Decomposition", IMF Working Paper, 00 (144).

Driver, R. et Westaway, P. F. (2005), "Concepts of Equilibrium Exchange Rates". Bank of England, Working paper, 248, 1-64.

Dufrenot G., Mathieu, L., Mignon, V. et Péguin-Feissolle, A. (2006),"Persistent Misalignments of the European Exchange Rates: Some Evidence from Nonlinear Cointegration", Applied Economics, 38(2): 203-229.

Faruqee, H. (1994), "Long-Run Determinants of the Real Exchange Rate - A Stock-Flow Perspective," IMF Working Papers 94/90.

Fernandez, F., Osbat, C. et Schnatz, B. (2001), "Determinants of the Euro Real Effective Exchange Rate: A BEER/PEER Approach." European Central Bank Working Paper.

Féroldi, M. et Sterdyniak, H. (1984), "De la Dynamique du Taux de Change: Variations sur un Theme de Dornbusch." Document de Travail de I'OFCE, 84 (10).

Gonzalo, J. et Granger, C. W. (1995), "Estimation of Common Long-Memory Components in Cointegrated Systems" Journal of Business Economics and Statistics, $13: 27-35$.

Houthakker, H.S. et Magee, S.P. (1969), "Income and Price Elasticities in World Trade" Review of Economics and Statistics, 51: 111-125.

Isard, P. et Faruqee, H. (1998), "Exchange Rate Assessment - Extensions of the Macroeconomic Balance Approach", IMF Occasional Paper, 167.

Krugman P. (1989), "Differences in Income Elasticities and Trends in Real Exchange Rates," NBER Working Papers 27 (61).

MacDonald, R. (1997), "What Determines Real Exchange Rates? The Long and Short of It," IMF Working Paper, 97 (21).

MacDonald, R. (2000), "Concepts to Calculate Equilibrium Exchange Rates: An Overview," Discussion Paper 3/00, Economic Research Group of the Deutsche Bundesbank.

Meese, R.A. et Rogoff, K. (1988), "What is Real ? The Exchange Rate - Interest Differential Relation over the Modern Floating Rate Period", Journal of Finance 42 (4): 933-947.

Prat, G. et Uctum, R. (2007), "Switching between Expectation Processes in the Foreign Exchange Market: a Probabilistic Approach using Survey Data," Review of International Economics, Blackwell Publishing, 15 (4) : 700-719. 
Siregar, R. et Rajan R. (2006), "Models of Equilibrium Real Exchange Rates Revisited: A Selective Review of the Literature," Centre for International Economic Studies Working Papers 2006-04, University of Adelaide, Centre for International Economic Studies.

Stein, J. L. (1994), "The Natural Real Exchange Rate of the Us-Dollar and Determinants of Capital Flows", Williamson J. (eds.), Estimating Equilibrium Exchange Rates, Washington, D.C., $133-176$.

Stein, J. L. et Allen, P. R. (1995), Fundamental Determinants of Exchange Rates, Clarendon Press.

Stein, J. L. (2002), "The Equilibrium Real Exchange Rate of the Euro: An Evaluation of Research," IFO Studien, 48 (3) : 349-81.

Usupbeyli, A. (2010) “Taux de Change Réels d'Equilibre et Dynamiques d'Ajustement Nonlinéaires : Une Application aux Données de la Turquie", Thèse de Doctorat.

Williamson, J. (1985), "The Exchange Rate System”, Policy Analyses in International Economics, vol. 5, Institute for International Economics, Washington D.C.

Williamson, J. (1994), "Estimates of FEERs", Williamson J. (eds), Estimating Equilibrium Exchange Rates, Washington, D.C., 177-244.

Williamson, J. et Miller, M. (1987), "Targets and Indicators: A Blueprint for the International Coordination of Economic Policy", Institute for International Economics, Washington DC: Policy Analyses in International Economics, No. 22.

Wren-Lewis, S., Westaway, P., Soteri, S. et Barrell, R. (1991), "Evaluating the UK's Choice of Entry Rate into the ERM", Manchester School, 59: 1-22.

Wren-Lewis, S. (1992), "On the Analytical Foundations of the Fundamental Equilibrium Exchange Rate," Hargreaves C. P. (eds.), Macroeconomic Modelling of the Long Run, (Alderhsot, Edward Elgar):75-94.

Wren-Lewis, S. et Driver, R. L. (1998), 'Real Exchange Rates for the Year 2000', Institute for International Economics, Washington DC: Policy Analyses in International Economics,54. 